\title{
List of Reviewers 2007
}

\author{
Eastern Economic Journal (2008) 34, 279. doi:10.1057/eej.2008.2
}

The Editors and Editorial Board wish to acknowledge the assistance of the following colleagues who reviewed articles during 2007 for their contributions of time and experience. Without their dedication, our work in publishing this Journal could not go forward.

\begin{tabular}{|c|c|c|}
\hline Monika AbuGhazaleh & Peter Deadman & Georgios Karras \\
\hline Scott Adams & Jakob de Haan & Mika Kato \\
\hline Richard Adelstein & Benedicte de La Briere & Dennis A. Kaufman \\
\hline Meherun Ahmed & Brigitte Desroches & Roger T. Kaufman \\
\hline Joshua Aizenman & Vaughan Dickson & Todd D. Kendall \\
\hline Gregory Amacher & Joseph A. DiMasi & Kristin Kleinjans \\
\hline Sudarat Anantochikul & John DiNardo & Jonathan Klick \\
\hline Gary Anderson & Anne-Celia Disdier & Thomas Konecny \\
\hline Soren Anderson & Emin Dokumaci & Peter James Lambert \\
\hline Charles Anderton & Konstantinos Drakos & John Lapp \\
\hline Christopher N. Annala & Scott David Drewianka & Blake LeBaron \\
\hline Peter Arcidiacono & Garey C. Durden & Oren Levin-Waldman \\
\hline Kartik B. Athreya & James A. Dunlevy & Fabrizio Lillo \\
\hline Kostas Axarloglou & Gary Dymski & Igor Livshits \\
\hline Robert U. Ayres & E. Woodrow Eckard & Mark C. Long \\
\hline Pio Baake & Susan Edelman & Sébastien Lotz \\
\hline Oscar Bajo-rubio & Jamie Emerson & Michael Lovell \\
\hline Jerome Ballet & Susan Louise Ettner & Mingshan Lu \\
\hline William Barber & Carolyn L. Evans & Stephanie Luce \\
\hline Debra A. Barbezat & Philip Fanara & Joseph Macri \\
\hline Reagan Baughman & Mohsen Fardmanesh & Wolfgang Maennig \\
\hline Giorgio Bellettini & Amy Farmer & John Marangos \\
\hline Mary Benedict & Doyne Farmer & Luigi Marengo \\
\hline David Berri & Amanda Jean Felkey & Fernand Martin \\
\hline Jeff Biddle & Angela Fertig & Joe Mason \\
\hline Robert Bifulco & Dennis Fok & Raymond Mataloni \\
\hline Jose Vicente Blanes-Cristobal & Duncan Foley & Bryan C, McCannon \\
\hline William M. Boal & Augustin Fosu & John M. McDowell \\
\hline Ronald Bodkin & James A. Freeman & Joseph P. McGarrity \\
\hline Allan Bolton & Renee Fry & Melayne McInnes \\
\hline Patricia Born & Delia Furtado & Jennifer M. Mellor \\
\hline Fernando Borraz & Todd Gabe & Debrah Meloso \\
\hline Anne D. Boschini & Robert F. Garnett, Jr. & Alexander Michaelides \\
\hline Yann Bramoulle & Robert Gazzale & Thomas Michl \\
\hline Dick Bryan & Ingo Geishecker & Richard A. Miller \\
\hline Kasey Buckles & Luis Alberiko Gil-Alana & Chris Milner \\
\hline Stefan Buhler & Gianfranco Giulioni & Andre Varella Mollick \\
\hline Joyce Burnette & Paul Glewwe & James Monks \\
\hline Meghan Busse & Marsha Goldfarb & Andrea Morone \\
\hline Matthieu Bussiere & Stephan Goetz & David Neumark \\
\hline Julie A. Carlson & Munisamy Gopinath & Edward Nissan \\
\hline John Caskey & Wayne Grove & Seth Norton \\
\hline Janet Ceglowski & Flore Gubert & Charles Okeahalam \\
\hline Latika Chaudhary & Alfred Volkmar Guender & Pia M. Orrenius \\
\hline Howard Chernick & Christopher Gunn & Ann L. Owen \\
\hline Robert Cherry & Catherine Hakim & Thomas Palley \\
\hline Georgios Chortareas & Mary Hansen & Randall Parker \\
\hline Shin-Yi Chou & Kjell Hausken & James Peach \\
\hline Hayley Helene Chouinard & Bernd Hayo & Jeffrey M. Perloff \\
\hline Abdulkadir Civan & Burkhard Hehenkamp & Joseph Persky \\
\hline Peter Colwell & John F. Helliwell & Inga Persson \\
\hline Adam Copeland & John Heywood & Paul Phillips \\
\hline Thomas Cosimano & Michael Hicks & Lisa Powell \\
\hline Tom Coupe & Alexander Hijzen & Lant Pritchett \\
\hline Theodore Covey & Vincent A. Hildebrand & Thomas Prusa \\
\hline German Gonzalo Creamer & John Hoag & Frederic L. Pryor \\
\hline Sean Crockett & Ed Hopkins & Jeffrey Racine \\
\hline David Cronin & Peter Howitt & Matiur Rahman \\
\hline Meredith Crowley & Masami Imai & Anoop Rai \\
\hline Patrick M. Crowley & Paul Isely & Peter Rangazas \\
\hline Ronald B. Davies & Tarun Jain & David Rapach \\
\hline John Davis & Marco Janssen & Inas Rashad \\
\hline Herbert Dawid & Sunil Kanwar & Kevin Rask \\
\hline
\end{tabular}

Farhad Rassekh

Kara M. Reynolds

John Rizzo

Eduardo Roca

James D. Rodgers

Brian Rogers

Elaina Rose

Alfonso Rosolia

Annalisa Russino

Dmitri Ryvkin

Elena Safirova

Allen R. Sanderson

Alicia C. Sasser

Yasuhiro Sato

Edward Scahill

Laurence S. Seidman

Mark Setterfield

Jorge M. Silva-Risso

Mark Skidmore

Frank Slesnick

Eric Smith

Christopher Snyder

Rohini Somanathan

Robert J. Sonora

Mark Stater

Faye Steiner

David W. Stewart

Leslie Stratton

Jan-Egbert Sturm

Michael T. Sumner

Steven Suranovic

Frank Thompson

John Thornton

Timo Tohmo

Mark Toma

Elichi Tomiura

Marc Tomljanovich

Marco Juri van der Leij

Roberto Veneziani

Arjan Verschoor

Martin Watts

Laurent Weill

Catherine J. Weinberger

Thomas Weisskopf

Jeanne Wendel

Jennie Wenger

Robert Whaples

Celia Whitchurch

Michelle J. White

Roger White

Jeannette Wicks-Lim

Allen Wilhite

Paul Conal Winters

Frances Woolley

Stephen Wu

Phanindra V. Wunnava

Bruce Wydick

Beiling Yan

Dean Yang

Peter A. Zaleski

Junfu Zhang 